\title{
Zugänge zum hinteren Beckenring
}

Wolfgang Lehmann

\section{Einleitung}

Die Wahl des Zugangs zum hinteren Beckenring ist vom Verletzungstyp, vom Ausmaß der Verletzung und von der Weichteilsituation abhängig. Man kann am hinteren Beckenring unterscheiden zwischen transiliakalen Verletzungen mit Frakturen im Bereich des Sakroiliakalgelenks (SI-Gelenks), reinen ligamentären sakroiliakalen Zerreißungen, Sakrumfrakturen und spinopelvinen Ausbruchverletzungen ( $\triangleright$ Abb. 1). Neben dem Verletzungsmuster ist für die Zugangswahl auch wichtig, ob eine Kompression neurologischer Strukturen, womöglich mit entsprechenden motorischen oder sensiblen Ausfällen, vorliegt.

Nach entsprechender Bildgebung, die immer eine CT beinhalten sollte, muss die Verletzung zunächst richtig klassifiziert werden. Je nach Verletzungsart können dann iliosakrale Schrauben, ilioiliakale Plattenosteosynthesen, spinopelvine Stabilisierungen allein oder als trianguläres Verfahren in Kombination mit einer iliosakralen Schraubenosteosynthese verwendet werden. Für die Zugänge zum Sakrum sind verschiedene Möglichkeiten beschrieben worden. Die iliosakrale Schraubenosteosynthese wird heute zumeist minimalinvasiv perkutan vorgenommen. Für die ilioiliakale Plattenosteosynthese werden beidseits Inzisionen neben den dorsalen Anteilen des Os ilium durchgeführt. Für die spinopelvinen Abstützungen oder auch triangulären Osteosynthesen, ob mit oder ohne Dekompression des Sakrums, hat sich in der eigenen Erfahrung die mediane Inzision als vorteilhaft erwiesen, da wir hier die wenigsten Weichteilkomplikationen gesehen haben.

Die Reposition einer dislozierten Iliosakralfugensprengung bzw. einer Sakrumfraktur und die Dekompression der neuralen Strukturen können dabei hoch anspruchsvoll sein und neurologische Ausfälle sind nach wie vor eines der häufigsten Probleme, gerade bei den schwer verschobenen Frakturen [9]. Ziel der vorliegenden Arbeit ist es, die einzelnen Zugänge und Repositionsmanöver darzustellen.

\section{Lagerung}

Bei den Operationen für den hinteren Beckenring wird der Patient unter Vollnarkose in Bauchlage auf einem völlig strahlendurchlässigen Carbontisch gelagert. Rollen werden entsprechend unter dem Thorax und dem Abdo- men positioniert, sodass das Becken frei bleibt. Um Lagerungsschäden, insbesondere Plexusschäden, zu vermeiden, muss sichergestellt sein, dass die Achselhöhlen frei sind und die Schultern nicht überstreckt werden. Wichtig ist, vor dem Abwaschen und Abdecken sicherzustellen, dass in allen wichtigen Projektionen, vor allem Inlet und Outlet, geröntgt werden kann. Es kann sinnvoll sein, für minmalinvasive Zugänge den Patienten vor der Operation abführen zu lassen, um Darmgasüberlagerungen im Röntgen zu vermeiden und eine bessere Übersicht zu haben. Wichtig ist, dass alle Röntgeneinstellungen exakt gewählt werden, um Fehllagen von Implantaten aufgrund schlechter Einstellungen bspw. in Inlet- oder Outlet-Darstellung zu vermeiden.

Merke

Vor dem Abdecken des Patienten prüfen, ob alle wichtigen Röntgenbilder sicher eingestellt werden können.

\section{Instrumente}

Für die Versorgung einer Beckenverletzung am hinteren Beckenring sind eine Reihe von Instrumenten unerlässlich. Dazu gehören Retraktoren, spezielle Beckenretraktoren, Repositionszangen, scharfe und stumpfe Einzinker, Kugelspieße mit und ohne Abstützschuh, Jungbluth-Zangen und Farabeuf-Zangen in unterschiedlichen Größen.

\section{Minimalinvasive Zugänge}

Minimalinvasiv ist vor allem die iliosakrale Schraubenosteosynthese. Sie ist sozusagen das „Arbeitspferd“ für die Stabilisierung des hinteren Beckenrings und die am häufigsten verwendete Osteosyntheseform. Zur beidseitigen Verschraubung bevorzugen wir die Bauchlage. Für einseitige Verschraubungen kann der Patient in Rückenlage gelagert werde. Er muss aber ausreichend an die Seite des Tisches gelagert werden, um auch genügend weit von dorsal den Eintrittspunkt wählen zu können.

Zu Beginn, insbesondere in der konventionellen Technik, wird im seitlichen Strahlengang der Eintrittspunkt für den Zieldraht aufgesucht ( $\bullet$ Abb. 2 ). Hierbei sollte der Startpunkt etwa in der Mitte vom 1. Kreuzbeinwirbel S I und dorsal des Spinalkanals liegen. Mit dem Zieldraht wird dann zunächst eben die äußere Wand der Darmbein- 

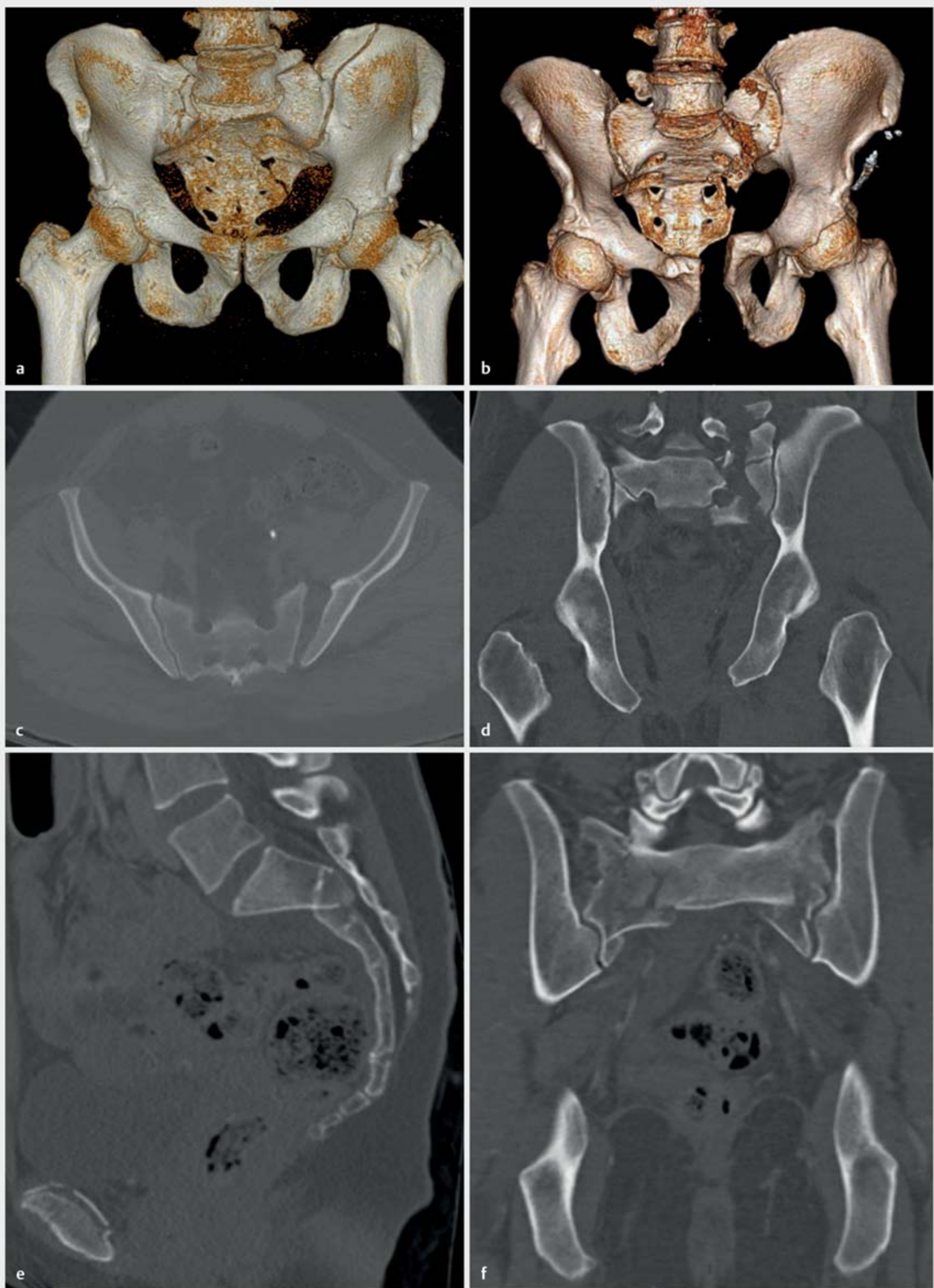

- Abb. 1 Beispiele unterschiedlicher Verletzungen des hinteren Beckenrings. a 3-D-Rekonstruktion im CT einer Fraktur des hinteren Beckenrings auf der linken Seite durch das Darmbein mit begleitender Fraktur am linken vorderen Schambeinast. b 3-D-Rekonstruktion im CT: linksseitige Fraktur des Sakrums mit Symphysensprengung. c Axiale CT-Schnitte einer reinen Sprengung der Iliosakralfuge. d Koronarer CT-Schnitt einer Sakrumfraktur mit Symphysensprengung. Das Becken ist auf der linken Seite schon nach oben disloziert. e und $\mathbf{f}$ Sagittales bzw. koronares CT-Bild einer spinopelvinen Ausrissverletzung. Hierbei ist die Wirbelsäule mit dem Kreuzbein vollständig vom Beckenring getrennt. 

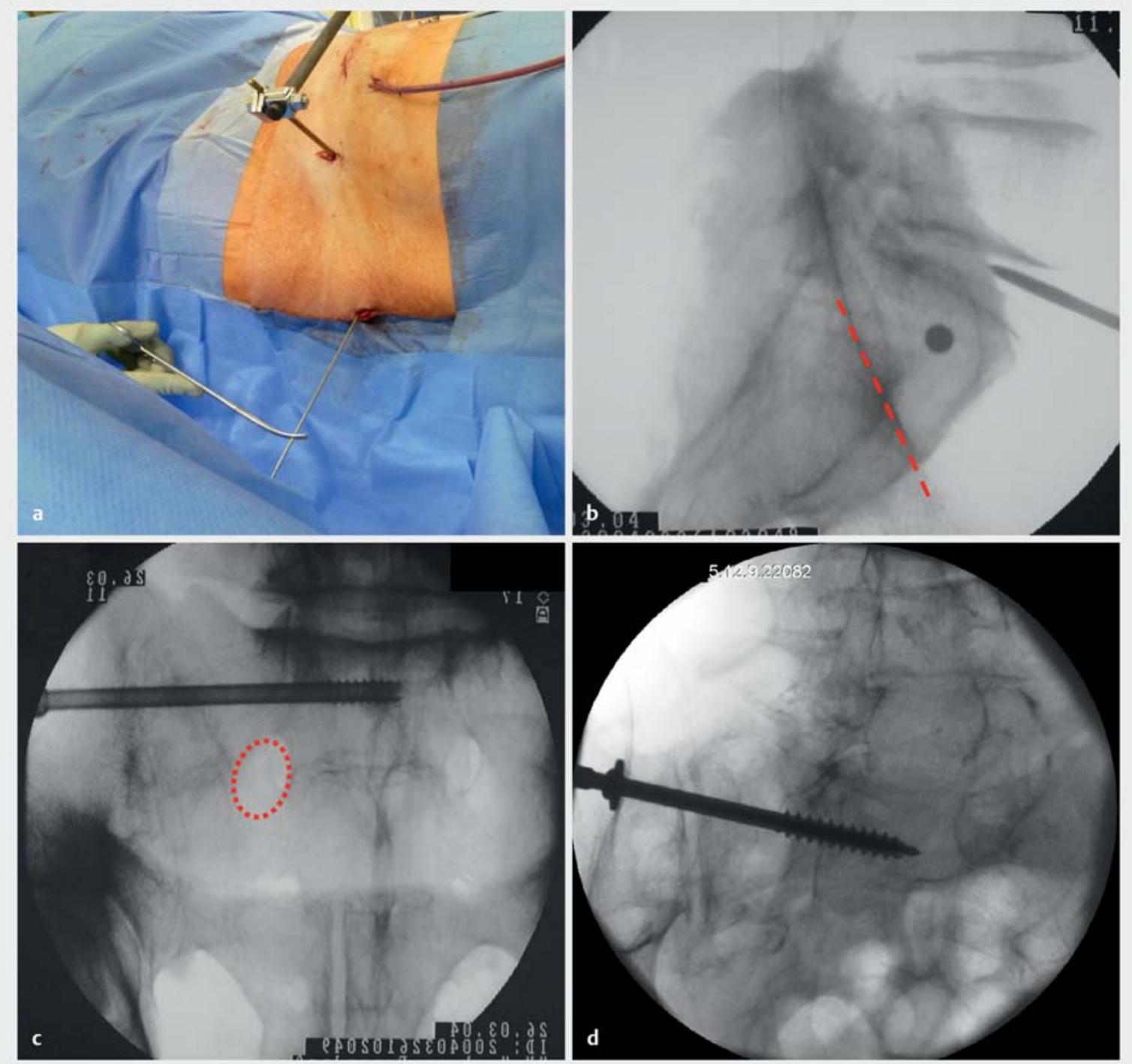

- Abb. 2 Minimalinvasive Verschraubung des hinteren Beckenrings. a Zunächst wird ein K-Draht angehalten, um die richtige Höhe im Seitbild zu erfassen. b Der Eintrittspunkt des K-Drahtes sollte hinter der ICD-Linie (ICD: iliac cortical density) liegen. Damit wird verhindert, dass der Draht vorne am Becken austritt. c Outlet-Aufnahme mit Blick auf die Neuroforamina (rot). $\mathbf{d}$ Inlet-Aufnahme mit Blick auf den 1. Kreuzbeinwirbel. Hier soll der Draht zentral zu liegen kommen.

schaufel penetriert und die korrekte Zieldrahtausrichtung in Relation zur „iliac cortical density“ (ICD), zum Neuroforamen SI und zur ventralen Begrenzung der Ala bzw. des Wirbelkörpers im Bildwandler überprüft [5]. Es ist wichtig, hinter dieser Linie mit dem Draht zu starten, da man so sicherstellt, dass der Draht nicht vorne durch die Darmbeinschaufel austritt und dann wieder in den 1 . Kreuzbeinwirbel eintritt und Nerven und Gefäße gefährdet. Nun wird der Draht bis in die Ala vorgebohrt und die Lage wiederum im Bildwandler kontrolliert. Im Falle der korrekten Platzierung wird der Zieldraht bis in den Wirbelkörper vorgebohrt und die nun resultierende Platzierung nochmals im Bildwandler überprüft. Nach dem
Überbohren des Zieldrahts mit einem 5,0-mm-Hohlbohrer erfolgt die definitive Schraubenplatzierung.

Die 2-D- oder 3-D-Navigation kann dabei hilfreich sein, die Schrauben schnell und korrekt ohne lange Durchleuchtungszeiten zu platzieren [3,4]. Als Implantate werden i.d.R. durchbohrte Spongiosaschrauben aus Titan mit 7,3 mm Durchmesser verwendet. Die Industrie bietet hierfür auch schon Schrauben an, die gleichzeitig zementiert werden können und bei denen die Unterlegscheiben mit den Schrauben verbunden sind. Idealerweise mit 32mm-Gewinde oder Vollgewinde, da diese gegenüber den 16-mm-Gewinden eine höhere biomechanische Stabilität bieten [6]. 


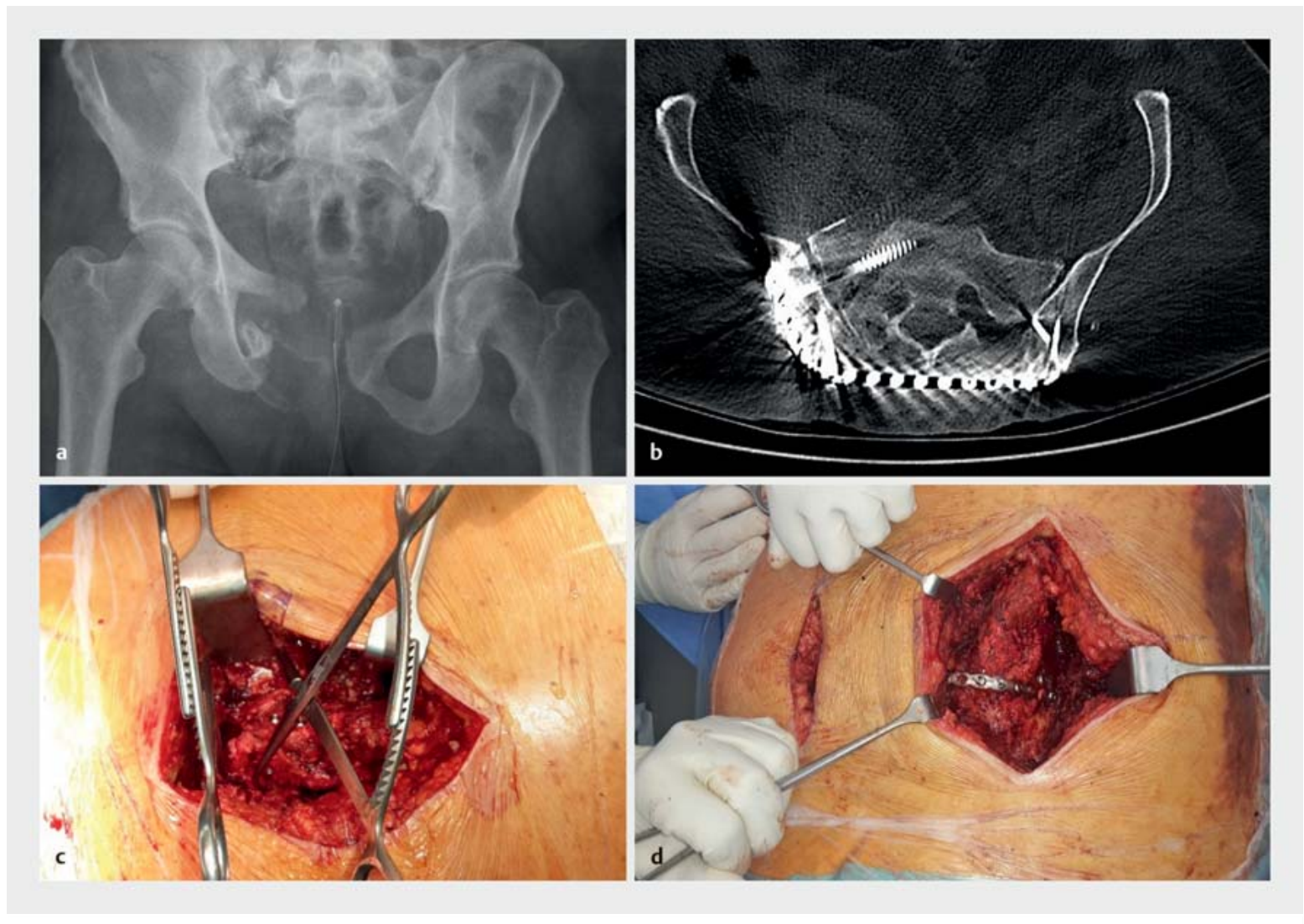

- Abb. 3 Bilder einer transiliakalen Plattenosteosynthese. a Präoperative Bilder einer dislozierten transiliakalen Fraktur. b CT axial postoperativ. c Reposition der transiliakalen Fraktur mit spitzen Repositionszangen. d Eingeschobene dorsale Plattenosteosynthese.

\section{Merke}

Für die iliosakrale Schraubenosteosynthese sollten entweder Vollgewindeschrauben oder Teilgewindeschrauben mit mindestens $32 \mathrm{~mm}$ Gewinde verwendet werden.

Wenn möglich, versuchen wir bei den hoch instabilen Verletzungen 2 Schrauben zu setzen, dies gelingt jedoch bei dem engen Korridor, der in anatomischen Präparaten nur durchschnittlich $27 \mathrm{~mm}$ breit und $21 \mathrm{~mm}$ weit ist, nicht immer [10]. Bei horizontal und vertikal instabilen Verletzungen sind 2 Schrauben biomechanisch stabiler und bieten eine höhere Sicherheit.

\section{Beidseitiger parailiakaler Zugang}

Der beidseitige Zugang entlang des hinteren Beckenkamms eignet sich insbesondere für die ilioiliakale Plattenosteosynthese. Der Vorteil dieser Osteosynthese liegt vor allem darin, dass Trümmerzonen des Sakrums gut überbrückt werden können und dann als „Distanzosteosynthesen“ verwendet werden, ohne eine Kompression mit Gefährdung nervaler Strukturen auszuüben [7]. Nach korrekter Lagerung werden die hinteren Darmbeinkämme auf beiden Seiten markiert. Die längs zu setzende
Hautinzision sollte nicht direkt über den Darmbeinkämmen verlaufen, damit ansonsten durch erhöhten Liegedruck in der postoperativen Phase auftretende Wundheilungsstörungen vermieden werden können. Dann wird die Faszie inzidiert und die Glutealmuskulatur von der Außenseite der Darmbeinschaufel abgeschoben [1]. Gleiches Vorgehen auf der Gegenseite. Bei dislozierten Frakturen eignet sich eine Schanz-Schraube in der Beckenschaufel, um die Fraktur zu reponieren und die Rotation und Kippung des Beckens auszugleichen. Unter Zug am Bein kann dann die endgültige Reposition erfolgen. Wenn die Stellung ideal ist, kann eine vorübergehende Stabilisierung mit einem gewindetragenden K-Draht sinnvoll sein, der eingebracht wird, wie für eine iliosakrale Schraube. Dann erfolgt die eigentliche Plattenosteosynthese, wobei diese idealerweise tief unten an den hinteren Darmbeinkämmen angebracht wird, um Weichteilschäden zu vermeiden. Zur Verwendung kommen 14bis 16-Loch-3,5er-Beckenrekonstruktionsplatten. Nachdem ein Kanal durch die Muskulatur bspw. mit einem großen Raspatorium gebahnt wurde, kann die Platte zur Gegenseite durchgeschoben werden ( $\triangleright$ Abb. 3). Gegebenenfalls kann eine Osteotomie auf Höhe der Crista mediana erfolgen, um die Platte möglichst dicht an das Sakrum heranzubringen. Dann wird zunächst auf einer Seite eine 
Schraube auf dem Kamm der Spina iliaca posterior inferior gesetzt und dann die Platte umgebogen auf die Außenseite des Os ilium. Es empfiehlt sich zu diesem Zeitpunkt, nochmals eine Röntgenkontrolle durchzuführen, um die waagerechte Ausrichtung der Platte sicherzustellen. Dann werden die restlichen Platten i.d.R. auf der zuerst präparierten Seite mit mindestens 3 bikortikal platzierten Schrauben durch das Os ilium besetzt [7].

Anschließend kann die Platte auf der Gegenseite noch mit einem Einzinkerhaken nach lateral gezogen werden, bevor sie mit einem Kugelspieß umgebogen wird, um eine gute Kompression auf den hinteren Beckenring zu erreichen. Nach dem Besetzen der Schrauben erfolgt der schichtweise Wundverschluss. Das Einlegen von RedonDrainagen auf jeder Seite kann helfen, postoperativ Hämatombildung bei kleineren Blutungen aus der abgelösten Glutealmuskulatur zu vermeiden.

\section{Direkte Zugänge zum Sakrum und der lliosakralfuge}

Die Größe des Zugangs ist abhängig vom Ausmaß der Stabilisierung. Lokale Plattenosteosynthesen bieten gegenüber den ilioiliakalen Plattenosteosynthesen den Vorteil, dass die intakte Iliosakralfuge dabei nicht tangiert wird. Lokale Plattenosteosynthesen sind nicht so stabil wie andere Osteosynthesen. Ziel der Versorgung ist die sorgfältige Dekompression des Spinalkanals und sichere Osteosynthese. Vor Beginn der Operation kann es sinnvoll sein, unter Durchleuchtungskontrolle durch Anheben der Beine eine Fraktur des Kreuzbeins zu reponieren. Insbesondere bei den Ausbruchverletzungen des Kreuzbeins ist die Osteosynthese zwischen der Wirbelsäule und dem Becken, die sog. spinopelvine Abstützung, die stabilste Art der Versorgung. Wenn zusätzlich noch eine iliosakrale Schraube eingebracht wird, spricht man von einer triangulären Osteosynthese [8]. Biomechanisch ist die trianguläre Fixation allen anderen Verfahren am hinteren Beckenring überlegen $[11,12]$. Der Zugang für diese Osteosynthesen kann als quere Inzision erfolgen [11] oder als Längsinzision. Aus eigener Erfahrung heraus verwenden wir die Mittellinieninzision, da wir hier weniger Weichteilkomplikationen sehen.

Die Längsinzision erfolgt über den Dornfortsätzen. Dann wird direkt bis auf den Knochen präpariert. Die Frakturen können mit einem Knochenspreizer aufgedehnt und mit scharfen Löffeln gesäubert werden. Um den Rückenmarkskanal bei einer Verlegung des Kanals freizuräumen, kann es notwendig sein, ergänzend zur Osteosynthese eine Laminektomie des Kreuzbeins vorzunehmen. Von zentral nach peripher können dann die Nervenwurzeln einzeln dargestellt werden.
Merke

CAVE: Bei schweren Verletzungen des Kreuzbeins können intraoperativ erhebliche Blutungen aus den Frakturflächen auftreten.

\section{Offene Reposition ligamentärer Iliosakralfugensprengungen}

Offene Repositionen der Iliosakralfugen können ausgehend von einer Mittelinieninzision oder weiter lateral erfolgen. Hier muss in Abhängigkeit von der größten Dislokation überlegt werden, ob die Versorgung von vorne oder von hinten erfolgen muss. Die lliosakralfugen können auch von vorne verplattet werden. Bei der Stabilisierung von hinten erfolgt, wie bei der ilioiliakalen Verplattung, die Inzision beginnend 1 bis $2 \mathrm{~cm}$ lateral der Spina iliaca posterior superior und posterior inferior [2]. Dabei kann die Inzision nach kaudal und kranial ausgedehnt werden. Mit gleicher Technik, wie bereits oben beschrieben, erfolgt dann wieder die subperiostale Ablösung des M. glutaeus maximus vom Os ilium. Im Bereich der SI-Fuge dorsal muss die Erector-trunci-Muskulatur nach medial abgeschoben werden, wobei hier als Grenze die Foramina sacralia zu berücksichtigen sind. Ein Knochenspreizer wird in das Iliosakralgelenk von dorsal eingesetzt, das Gelenk aufgeweitet und unter Sicht sorgfältig débridiert und gespült. Hierfür verwenden wir gerne auch eine gepulste Lavage, um Hämatome etc. vollständig auszuspülen. Der Spreizer wird entfernt und die Darmbeinschaufel muss nun mit ihrem Gelenkanteil anatomisch in die Gelenkfläche des Sakrums eingepasst werden. Die Repositionskontrolle kann radiologisch in der Inlet- und Outlet-Aufnahme oder digital durch die Incisura ischiadica major erfolgen. Die temporäre Stabilisierung erfolgt mit einer großen Weber-Repsitionszange, die zwischen der Außenseite des Os ilium und nach kurzstreckiger Präparation - der Crista mediana eingehängt wird. Bei sehr adipösen Patienten haben wir auch schon eine Beckenzwinge verwendet, die wir in Bauchlage angelegt haben, um ausreichende Kompression auf die Iliosakralfuge zu bekommen ( Abb. 4).

Nach der Reposition des SI-Gelenks erfolgt die Fixierung mit einem gewindetragenden K-Draht der Dicke 2,8 mm, über den dann auch die kanülierte Schraube eingebracht werden kann. Es schließt sich die sorgfältige Kontrolle der Reposition in der Inlet-, Outlet- und a.-p. Ansicht an. Zur Qualitätsverbesserung hat sich die intraoperative CTDiagnostik mit einem 3-D-Bildwandler sehr bewährt, da es so möglich wird, das lliosakralgelenk sehr genau darzustellen. Die Stabilisierung erfolgt dann mit 1-2 iliosakralen Schrauben.

Merke

Die intraoperative Darstellung mit einem 3-D-Bildwandler nach der Reposition und vor der definitiven Stabilisierung hilft, Fehlstellungen zu vermeiden. 

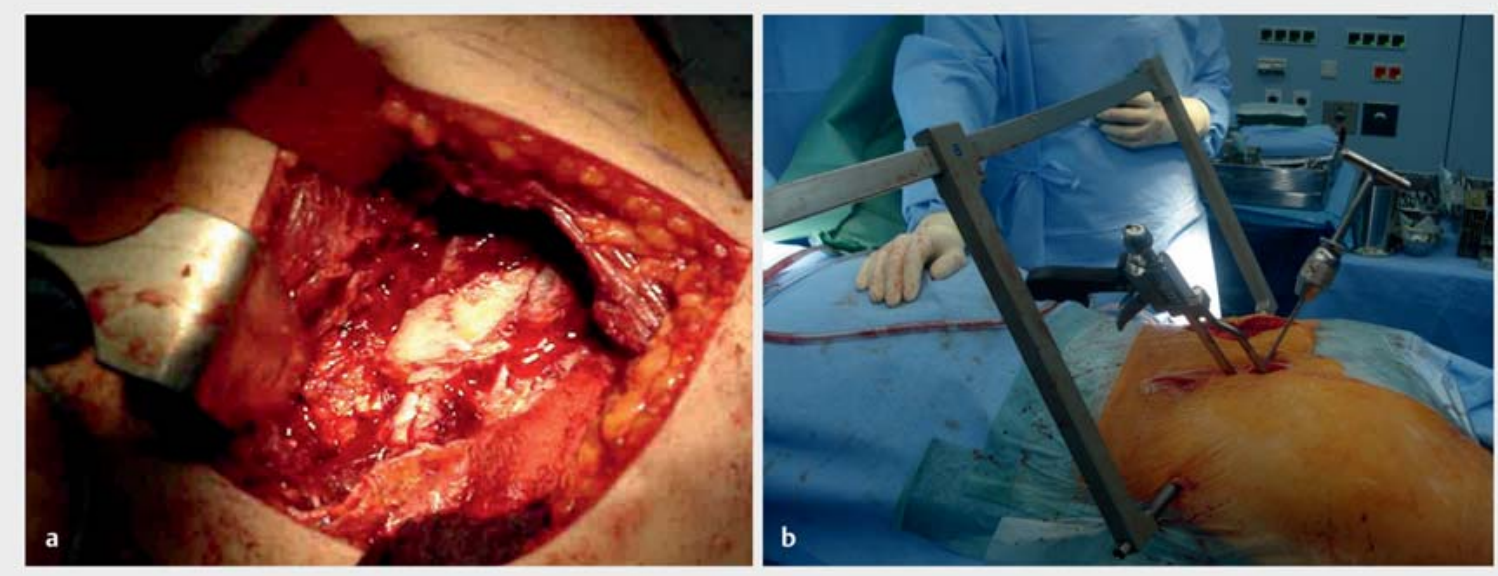

- Abb. 4 a Ausgedehnte Fraktur des Sakrums. b Um Frakturen des hinteren Beckenrings gut zu reponieren, kann auch eine Beckenzwinge in Bauchlage hilfreich sein. Weitere Repositionshilfen sind Schanz-Schrauben in der Darmbeinschaufel oder auch eine koaxiale Zange, die am Sakrum eingehängt werden kann.

\section{Technik der lumbopelvinen Stabilisierung}

Für die lumbopelvine Stabilisierung wird der Zugang über eine Mittellinieninzision entsprechend erweitert, sodass die Pedikel von L V bzw. L IV dargestellt werden. Eine weitere Möglichkeit eines kleinen Zugangs ist das direkte Splitting der Muskulatur über den Wiltse-Zugang [13]. Grundsätzlich verwenden wir hier auch die Mittellinieninzision. Gerade bei schweren knöchernen Verletzungen kann es zu Morel-Lavallée-Verletzungen kommen ( $\vee$ Abb. 5). Dabei handelt es sich um weitreichende Ablösungen des Unterhautfettgewebes von der Faszie. Diese Verletzungen sind mit einem sehr hohen Infektionsrisiko verbunden. Sollte die Beckenverletzung mit einer MorelLavallée-Verletzung einhergehen, ist es sinnvoll, die Wunde nach der Versorgung 48 Stunden später noch einmal zu spülen oder einen Vakuumverband einzulegen.

\section{Merke}

Morel-Lavallée-Verletzungen gehen mit einem hohen Infektionsrisiko einher.

Nachdem die Pedikelschrauben gesetzt sind, erfolgt das Setzen der Iliumschrauben, was die gebräuchlichste Technik darstellt, oder lateral der Fraktur in das Sakrum selbst. Die Schrauben in der Ala sacralis können bikortikal gesetzt werden, um gerade im osteoporotischen Knochen einen festen Halt zu gewährleisten. Aufgrund der ventralen Gefäße dürfen diese Schrauben die ventrale Kortikalis nicht wesentlich überragen. Die Lage dieser Schrauben kann mit der Inlet-Projektion überprüft werden. Um die Schraube korrekt zu platzieren, muss der „Korridor“ im Bildwandler korrekt eingestellt werden ( $\bullet$ Abb. 5). Hierzu dient die Obturator-Outlet-Aufnahme. Ziel ist dabei, mit einer möglichst langen und dicken Schraube (ca. 7$8 \mathrm{~mm}$ Dicke und 90-110 mm Länge) eine feste Verankerung zwischen den beiden Kortikalisblättern des Os ilium zu erreichen. Dabei können prinzipiell alle langen Pedikelschrauben verwendet werden. Sehr scharfe, selbstschneidende Schrauben haben den Nachteil, dass sie sich nicht ihren Weg zwischen den beiden Kortikaliswänden suchen, sondern dazu neigen, nach lateral auszubrechen und so eine Fehllage produzieren.

Bei erheblich dislozieren Frakturen eignet sich das USSSystem von Synthes, sodass über die hervorstehenden Stangen direkt reponiert werden kann. Wenn es gelingt, die Reposition vorher mittels einer Weber-Klemme zu erreichen, kann mit polyaxialen Schrauben allerdings das Setzen des Längsverbinderstabs deutlich vereinfacht sein. Wichtig ist, darauf zu achten, dass die Backen des verwendeten Fixateurs nicht zu prominent dorsal über dem Os ilium auftragen, da diese schwere Weichteilprobleme verursachen können. Gegebenenfalls muss hier bei sehr schlanken Patienten im hinteren Beckenkamm eine Nut geschaffen werden, um den Kopf der Schraube zu versenken.

\section{Fazit}

Zugänge zum hinteren Beckenring sind technisch einfach. Die Herausforderung ist vielmehr, die Diagnose exakt zu stellen und die Verletzung korrekt zu klassifizieren. Dadurch ergibt sich die operative Vorgehensweise. Die Reposition kann manchmal bei sehr verschobenen Frakturen anspruchsvoll sein. Gerade bei den horizontal und vertikal instabilen Verletzungen wird meistens zuerst von dorsal stabilisiert. Die begleitenden Weichteilverlet- 

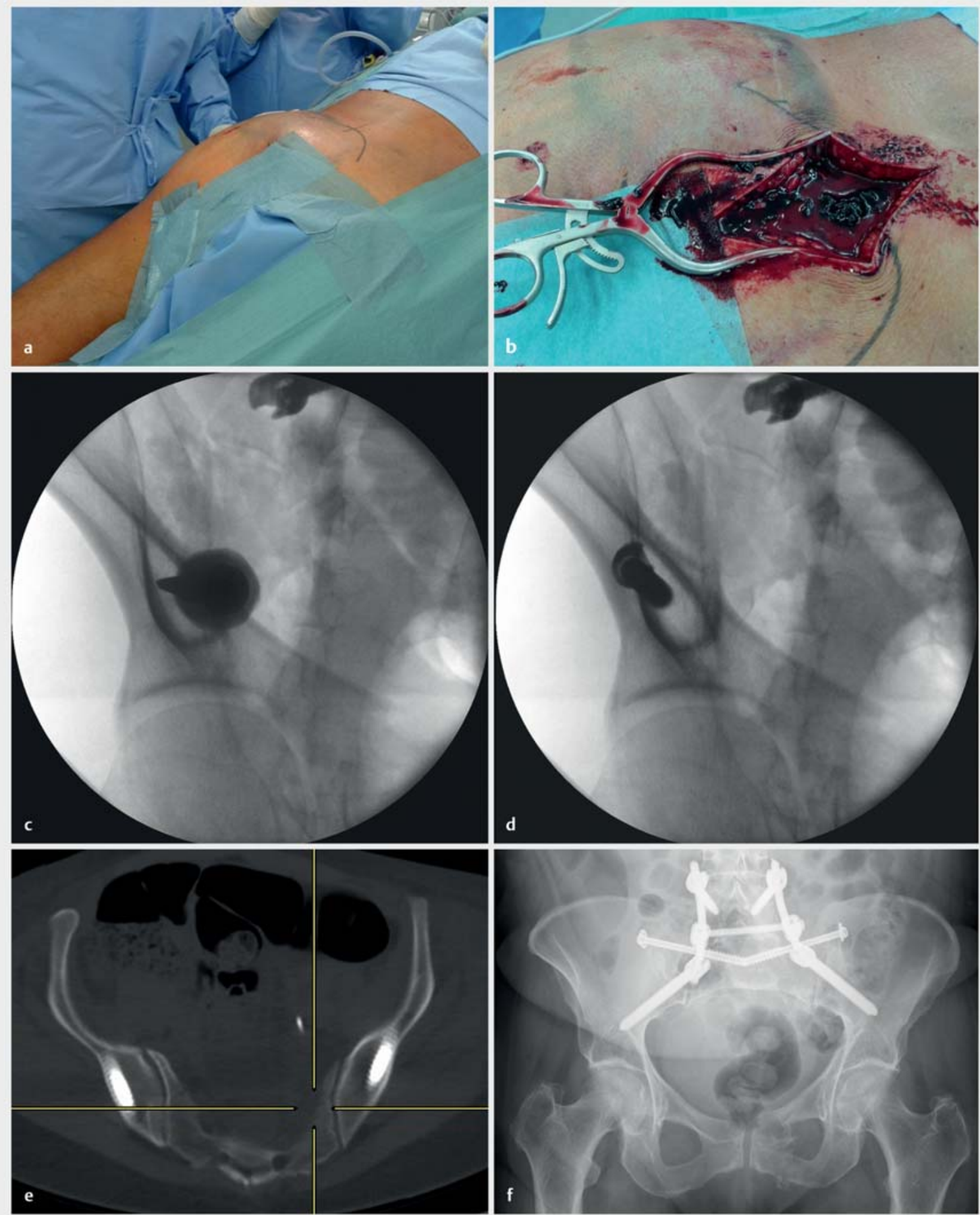

- Abb. 5 a Lagerung in Bauchlage. Gegebenenfalls muss ein Bein, in manchen Fällen auch beide Beine, beweglich abgedeckt werden. b Nach Hautinzision zeigt sich ein erhebliches Hämatom subkutan. Dies ist das Bild einer Morel-Lavalée-Verletzung. c und d In der Obturator-Outlet-Aufnahme sieht man den Kanal, in den die langen Schrauben für die spinopelvine Abstützung in die Darmbeinschaufel eingedreht werden. e Im CT-Schnitt erkennt man die korrekte Lage der langen Schrauben. $f$ Beckenübersicht nach triangulärer Osteosynthese des hinteren Beckenrings. 
zungen am hinteren Beckenring müssen bei der Zugangswahl ebenfalls in die Überlegung mit eingeschlossen werden. Ziel des Eingriffs ist die Wiederherstellung der Form und Funktion des hinteren Beckenrings, die es dem Patienten ermöglicht, frühzeitig wieder voll zu belasten.

Interessenkonflikt

Der Autor gibt an, dass kein Interessenkonflikt besteht.

\section{Autorinnen/Autoren}

\section{Univ.-Prof. Dr. med. Wolfgang Lehmann}

Direktor der Klinik für Unfallchirurgie, Orthopädie und Plastische Chirurgie, Universitätsmedizin Göttingen

\section{Korrespondenzadresse}

Univ.-Prof. Dr. med. Wolfgang Lehmann

Klinik für Unfallchirurgie, Orthopädie und Plastische Chirurgie Universitätsmedizin Göttingen, Georg-August-Universität Robert-Koch-Straße 40

37099 Göttingen

Tel.: 05 51/39-22462 (-22459)

Fax: 05 51/39-8991

wolfgang.lehmann@med.uni-goettingen.de

\section{Literatur}

[1] Bahmann D, Larndorfer R, Krappinger D et al. Stabilization of the posterior pelvic ring with a slide-insertion plate. Oper Orthop Traumatol 2007; 19: 16-31

[2] Borrelli J jr., Koval KJ, Helfet DL. Operative stabilization of fracture dislocations of the sacroiliac joint. Clin Orthop Relat Res 1996; (329): 141-146

[3] Briem D, Linhart W, Lehmann W et al. Computer-assisted screw insertion into the first sacral vertebra using a threedimensional image intensifier: results of a controlled experimental investigation. Eur Spine J 2006; 15: 757-763
[4] Briem D, Rueger JM, Begemann PG et al. [Computer-assisted screw placement into the posterior pelvic ring: assessment of different navigated procedures in a cadaver trial]. Unfallchirurg 2006; 109: 640-646

[5] Briem D, Windolf J, Rueger JM. [Percutaneous, 2D-fluoroscopic navigated iliosacral screw placement in the supine position: technique, possibilities, and limits]. Unfallchirurg 2007; 110: 393-401

[6] Gansslen A. [Biomechanical principles for treatment of osteoporotic fractures of the pelvis]. Unfallchirurg 2010; 113: 272280

[7] Lehmann W, Grossterlinden L, Rueger JM. [Posterior approaches to the pelvic ring]. Unfallchirurg 2013; 116: 205-212

[8] Lehmann W, Hoffmann M, Briem D et al. Management of traumatic spinopelvic dissociations: review of the literature. Eur J Trauma Emerg Surg; 38: 517-524

[9] Moon CN, Merkle PF. A level one trauma center's experience with the posterior approach to the pelvis. Orthopedics 2002; 25: $159-162$

[10] Noojin FK, Malkani AL, Haikal L et al. Cross-sectional geometry of the sacral ala for safe insertion of iliosacral lag screws: a computed tomography model. J Orthop Trauma 2000; 14: 31-35

[11] Schildhauer T A, Josten C, Muhr G. Die trianguläre Osteosynthese instabiler Sakrumfrakturen. Operat Orthop Traumatol 2001; 13: 27-42

[12] Schildhauer TA, Ledoux WR, Chapman JR et al. Triangular osteosynthesis and iliosacral screw fixation for unstable sacral fractures: a cadaveric and biomechanical evaluation under cyclic loads. J Orthop Trauma 2003; 17: 22-31

[13] Wiltse LL, Bateman JG, Hutchinson RH et al. The paraspinal sacrospinalis-splitting approach to the lumbar spine. J Bone Joint Surg Am 1968; 50: 919-926

Bibliografie

DOI https://doi.org/10.1055/s-0043-125353

OP-JOURNAL 2018; 34: 40-47 @ Georg Thieme Verlag KG Stuttgart · New York ISSN 0178-1715 